\title{
An SOS-QE Approach to Nonlinear Gain Analysis
}

\author{
Hiroyuki Ichihara† and Hirokazu Anaił§
}

\begin{abstract}
A hybrid computational method between sum of squares (SOS) and quantifier elimination (QE) is discussed for a problem of nonlinear gain analysis. A computational procedure is proposed to be applied to nonlinear polynomial systems. The procedure takes two major parts: the first part is searching a structure in the gain functions while the second part reveals all the gain functions in the structure. These tasks are assigned to the SOS and QE approaches, respectively. In order to find an accurate system performance, the gain functions are optimized in the procedure. The proposed analysis procedure is illustrated for a few systems.
\end{abstract}

\section{INTRODUCTION}

Symbolic methods in control engineering have attracted considerable attention in recent years. In particular, quantifier elimination (QE) has been studied with regard to its applications in robust control [1], [2], [3] and model predictive control [4] based on multi-parametric optimization [5]. As the name suggests, QE methods eliminate quantifiers in a well-formed formula in the first order theory of real closed field. A powerful algorithm is cylindrical algebraic decomposition (CAD)[6]. Since the computational cost of $\mathrm{QE}$ is often high, several investigations have been conducted for special classes of $\mathrm{QE}$ problems.

Nowadays additional effort is still required to remove heuristic procedures in engineering problems. An approach resolving this issue is based on the combined use of numerical and symbolic methods. One successful combination, the method of sum of squares (SOS) [7], [8], has already been proposed. This method is employed to solve the problems consisting of polynomial inequalities with universal quantifiers, where it eliminates the quantifiers to reduce the formula to a semidefinite programming (SDP) problem [9]. As the success of SOS greatly depends on solving the SDP problem, it is often categorized as a numerical method. Although both QE and SOS are tools in real algebraic geometry, SOS loses some remarkable characteristics in $\mathrm{QE}$, such as exactness in nonconvex optimization.

In this paper, we consider the problem of computing a nonlinear gain function [10], [11] for a class of dynamical systems. The notion of input-to-state stability (ISS) is strongly related to the concept of nonlinear gain. ISS with nonlinear gain is essential because the stability of nonlinear systems cannot be simply classified as asymptotic or exponential stability. For a system, the existence of an ISSLyapunov function with a nonlinear gain function means that the system has ISS. There exist certain numerical methods

†School of Science and Technology, Meiji University, Kawasaki 2148571, Japan. ‡Design Innovation Laboratory, Fujitsu Laboratories Ltd., Kawasaki 211-8588, Japan. §Graduate School of Mathematics, Kyushu University, Fukuoka 819-0395, Japan. for checking the Hurwitz stability of linear systems, such as eigenvalue analysis. Similarly, computational methods that employ SOS have been proposed for checking the ISS of polynomial nonlinear systems [12]. It is possible to minimize a linear gain value, or $\mathcal{L}_{2}$ gain value [9], using SDP. However, as far as we know, the method of SOS cannot minimize a nonlinear gain function because such a function includes multiple parameters that require optimization. In other words, numerical methods only ensure the existence of a nonlinear gain function. On the other hand, the $\mathrm{QE}$ approach can potentially perform minimization of such a function provided that all sets of the gain functions are given. That is, the relationship between coefficients on each monomial in the gain functions must be given. To compensate for the shortcoming of the QE approach, i.e., the very long computational time, it is desirable that a feasible structure of the gain function is known. The SOS approach is a very effective way of finding such a feasible structure. This is a key point of combination of SOS and QE. From this viewpoint, we have already proposed a combination of SOS and QE [13], in which the class of the gain functions is restricted to a form. In this paper, we propose a new analysis procedure that treats a wider class of the gain functions. In addition, we provide an application to the ISS small-gain theorem [14] that ensures global asymptotic stability (GAS) of the interconnected systems including design parameters.

The rest of the paper is organized as follows: Section II is devoted to a short introduction of nonlinear gain analysis. In section III we briefly review two tools in real algebraic geometry: SOS and QE. A computational method that employs SOS to find a nonlinear gain function is discussed in section IV. A method for assembling all the gain functions through QE is explained in section V. In section VI, a procedure to obtain a minimum gain function is proposed and demonstrated. Finally, we conclude the paper and provide additional remarks in section VII.

Notation: For a vector $x \in \mathbb{R}^{n},\|x\|$ represents the Euclidean norm of $x$. "o" represents the composition of maps such that $\alpha_{1} \circ \alpha_{2}(r)=\alpha_{1}\left(\alpha_{2}(r)\right)$. For an $m \times n$ matrix $A$ of real elements, the induced norm of $A$ is defined by $\|A\|=$ $\sup _{x \neq 0}(\|A x\| /\|x\|)$. For a symmetric matrix $Q, Q \succ(\succeq) 0$ represents positive definite (semidefinite).

Throughout the paper, we use the following comparison functions:

Definition 1.1: [15] A continuous function $\alpha:[0, \infty) \longrightarrow$ $[0, \infty)$ is said to belong to class $\mathcal{K}$ if it is strictly increasing and $\alpha(0)=0$. It is said to belong to class $\mathcal{K}_{\infty}$ if $\alpha(r) \longrightarrow \infty$ as $r \longrightarrow \infty$. 
Definition 1.2: [15] A continuous function $\beta:[0, \infty) \times$ $[0, \infty) \longrightarrow[0, \infty)$ is said to belong to class $\mathcal{K} \mathcal{L}$ if, for each fixed $s$, the mapping $\beta(r, s)$ belongs to class $\mathcal{K}$ with respect to $r$ and, for fixed $r$, the mapping $\beta(r, s)$ is decreasing with respect to $s$ and $\beta(r, s) \longrightarrow 0$ as $s \longrightarrow \infty$.

Lemma 1.1: [15] Let $\alpha$ and $\beta$ be class $\mathcal{K}_{\infty}$ functions. The inverse of $\alpha$ is denoted by $\alpha^{-1}$. Then $\alpha^{-1}$ is defined on $[0, \infty)$ and belongs to class $\mathcal{K}_{\infty}$, and $\alpha \circ \beta$ belongs to class $\mathcal{K}_{\infty}$

\section{NONLINEAR GAIN ANALYSIS}

Consider the system

$$
\dot{x}(t)=f(x(t), w(t)), x(0)=x_{0}, t \geq 0
$$

where $x \in \mathbb{R}^{n}$ is the state, $w \in \mathbb{R}^{p}$ is an exogenous input, $f: \mathbb{R}^{n} \times \mathbb{R}^{p} \rightarrow \mathbb{R}^{n}$ is a continuously differentiable function. Suppose that $f(0,0)=0$ and $w(t)$ is a piecewise continuous, bounded function in $t$ for all $t \geq 0$. For the system (1), we introduce the following concept of stability.

Definition 2.1: [10] The system (1) is said to be inputto-state stable if there exists a class $\mathcal{K} \mathcal{L}$ function $\beta$ and a class $\mathcal{K}$ function $\gamma$, called a nonlinear gain function, such that for any initial state $x(0)$ and any bounded input $w(t)$, the solution $x(t)$ exists for all $t \geq 0$ and satisfies

$$
\|x(t)\| \leq \beta\left(\left\|x_{0}\right\|, t\right)+\gamma\left(\sup _{\tau \in[0, t]}\|w(\tau)\|\right) .
$$

Definition 2.2: [10] A continuously differentiable $V$ : $\mathbb{R}^{n} \rightarrow \mathbb{R}$ is called an ISS-Lyapunov function for the system (1) if there exist class $\mathcal{K}_{\infty}$ functions $\alpha_{i}, i=1,2$, class $\mathcal{K}$ functions $\mu$ and $\rho$ such that

$$
\begin{aligned}
& \alpha_{1}(\|x\|) \leq V(x) \leq \alpha_{2}(\|x\|) \quad \forall x \in \mathbb{R}^{n} \\
& \frac{\partial V}{\partial x} f(x, w) \leq-\mu(\|x\|) \quad \forall\|x\| \geq \rho(\|w\|) .
\end{aligned}
$$

From the definition above, one can obtain a nonlinear gain function $\gamma(r)$ as $\alpha_{1}^{-1} \circ \alpha_{2} \circ \rho(r)$ [10].

Lemma 2.1: [10] The system (1) is input-to-state stable with $\gamma$ if and only if there exist a continuously differentiable function $V: \mathbb{R}^{n} \rightarrow \mathbb{R}$, class $\mathcal{K}_{\infty}$ functions $\alpha_{i}, i=1,2,3$, a class $\mathcal{K}$ function $\sigma$ such that (3) and

$$
\frac{\partial V}{\partial x} f(x, w) \leq \sigma(\|w\|)-\alpha_{3}(\|x\|)
$$

for all $(x, w) \in \mathbb{R}^{n} \times \mathbb{R}^{p}$. Then $\gamma(r)=\alpha_{1}^{-1} \circ \alpha_{2} \circ \alpha_{3}^{-1} \circ k \sigma(r)$ for some $k>1$.

If $V$ is a $\mathcal{K}_{\infty}$ function, it is possible to choose $\alpha_{1}$ and $\alpha_{2}$ such as $\alpha_{1}=\alpha_{2}$ in (3). Then one can omit the condition (3) in Lemma 2.1 to ensure the system ISS.

Corollary 2.1: The system (1) is input-to-state stable with $\gamma$ if there exist a continuously differentiable and class $\mathcal{K}_{\infty}$ function $V: \mathbb{R}^{n} \rightarrow \mathbb{R}$, a class $\mathcal{K}_{\infty}$ function $\alpha_{3}$, a class $\mathcal{K}$ function $\sigma$ such that (5) for all $(x, w) \in \mathbb{R}^{n} \times \mathbb{R}^{p}$. Then $\gamma(r)=\alpha_{3}^{-1} \circ k \sigma(r)$ for some $k>1$.
From this point, we will use Corollary 2.1 to discuss nonlinear gain analysis. In this paper, minimization of the nonlinear gain function is defined by the following:

$$
\begin{gathered}
\underline{\gamma}(r)=\min _{\alpha_{3} \in \mathcal{K}_{\infty}, \alpha_{4} \in \mathcal{K}} \alpha_{3}^{-1} \circ \alpha_{4}(r) \text { for each } r>0 \\
\text { s.t. }(\forall x \forall w)[(5)]
\end{gathered}
$$

Then all the functions $\gamma$ satisfying $\gamma(r)>\gamma(r)$ for each $r>$ 0 are nonlinear gain. In particular, we call $\gamma$ the minimum gain function since it represents the minimum function of $\gamma$ for each $r>0$.

Consider the feedback interconnected system

$$
\begin{aligned}
& \dot{x}_{1}(t)=f_{1}\left(x_{1}(t), x_{2}(t)\right) \\
& \dot{x}_{2}(t)=f_{2}\left(x_{1}(t), x_{2}(t), w(t)\right)
\end{aligned}
$$

where $x_{1} \in \mathbb{R}^{n_{1}}, x_{2} \in \mathbb{R}^{n_{2}}, f_{1}: \mathbb{R}^{n_{1}} \times \mathbb{R}^{n_{2}} \rightarrow \mathbb{R}^{n_{1}}$ and $f_{2}: \mathbb{R}^{n_{1}} \times \mathbb{R}^{n_{2}} \times \mathbb{R}^{p} \rightarrow \mathbb{R}^{n_{2}}$ are continuously differentiable functions. Suppose that $f_{1}(0,0)=0$ and $f_{2}(0,0,0)=0$. Then the following lemma is referred to as the ISS smallgain theorem.

Lemma 2.2: [14] Let $\gamma_{1}, \gamma_{2} \in \mathcal{K}$ be nonlinear gain functions of the system (6) with $x_{2}$ as input, and the system (7) with $\left(x_{1}, w\right)$ as input, respectively. If the following relation

$$
\gamma_{1} \circ \gamma_{2}(r)<r \quad \forall r>0
$$

holds, then the interconnected system (6) and (7) is ISS with respect to state $\left(x_{1}, x_{2}\right)$ and input $w$.

Remark 2.1: If $w=0$ in Lemma 2.2, then the origin $\left(x_{1}, x_{2}\right)=(0,0)$ of the interconnected system (6) and (7) is 0 -GAS.

\section{Tools in Real Algebraic Geometry}

In this section, we introduce two computational tools in real algebraic geometry: One is SOS, which is available for checking positive semidefiniteness of polynomials through $\mathrm{SDP}$, and the other one is $\mathrm{QE}$, which can treat first-order formulas based on CAD algorithms.

\section{A. SOS}

Let us consider a polynomial with degree $2 d$ as

$$
p(x)=\sum_{\beta \in \mathcal{B}} p_{\beta} x^{\beta}
$$

where $x \in \mathbb{R}^{n}, x^{\beta}=x_{1}^{\beta_{1}} \cdots x_{n}^{\beta_{n}}$ and

$$
\mathcal{B}=\left\{\beta \in \mathbb{R}^{n}: \sum_{i} \beta_{i} \leq 2 d\right\} .
$$

Now we assume that $p(x)$ is nonnegative (or positive semidefinite) for all $x$. A deceptively simple sufficient condition for $p(x)$ to be nonnegative everywhere is given by the existence of an SOS decomposition:

$$
p(x)=\sum_{i} q_{i}^{2}(x)
$$

where each $q_{i}$ is a polynomial. It is clear that if a given polynomial $p(x)$ can be written in the form above, then it is nonnegative for all $x$. A polynomial that has SOS decomposition can be rewritten in a quadratic form as $s(x)=$ $z(x)^{T} Q z(x)$ where $Q$ is a semidefinite matrix and $z(x)$ is 
a vector of monomials in $x$ [7], [8]. Here, finding $Q$ that satisfies $p(x)=s(x)$ for all $x$ is called an SOS problem. A certification that ensures $Q \succeq 0$ depends on the feasibility of an SDP problem such as

$$
\exists Q(\succeq 0) \text { s.t. } \operatorname{tr}\left(A_{\beta} Q\right)=p_{\beta} \forall \beta \in \mathcal{B}
$$

where $A_{\beta}$ satisfies $z z^{T}=\sum_{\beta \in \mathcal{B}} A_{\beta} x^{\beta}$. If the problem (10) is feasible, then $p(x)$ is an SOS polynomial and is thus nonnegative for all $x$. In some families, nonnegative polynomials are always SOS ones. The first family is the case of univariate polynomials $(n=1)$. The second one is in quadratic form $(d=1)$. The last one is in quartic form in two variables $(d=2, n=2)$. The other families of polynomials admit a gap between non-negativeness and SOS.

In the case where the individual coefficients in a polynomial linearly include some unknown parameters, one can also employ SDP to find such parameters that make the polynomial SOS. Consider a polynomial with degree $2 d$ as

$$
p(x ; c)=\sum_{\beta \in \mathcal{B}} p_{\beta}(c) x^{\beta}
$$

where $p_{\beta}(c)=p_{\beta}(0)+\sum_{j=1}^{n_{c}} c_{j} p_{\beta}^{(j)}$ and $c \in \mathbb{R}^{n_{c}}$ is an unknown vector. By letting $p(x ; c)=s(x)$, one can obtain an SDP problem:

$$
\exists Q(\succeq 0) \exists c \in \mathbb{R}^{n_{c}} \text { s.t. } \operatorname{tr}\left(A_{\beta} Q\right)=p_{\beta}(c) \forall \beta \in \mathcal{B} .
$$

There are a few SOS parsers on Matlab, such as SOSTOOLS [16] and YALMIP [17] which generate an SDP problem from an SOS formulation. General SDP solvers such as SeDuMi [18] and SDPT3 [19] on Matlab can handle both types of SDP problems (10) and (11). The method of nonnegativity check through SOS and SDP is an efficient and convenient tool in control engineering [20].

\section{B. $Q E$}

Many mathematical and industrial problems can be naturally translated to formulas consisting of polynomial equations, inequalities, quantifiers $(\forall, \exists)$ and Boolean operators $(\wedge, \vee, \neg, \rightarrow, e t c)$. Such formulas construct sentences in the first-order theory of real closed fields and are called firstorder formulas. We denote these formulas by $f_{i}(X, U)$, $i=1,2, \ldots, t$, polynomials in $X, U$ over the reals $\mathbb{R}$, where $X=\left(x_{1}, \ldots, x_{n}\right) \in \mathbb{R}^{n}$ is a set of quantified variables and $U=\left(u_{1}, \ldots, u_{m}\right) \in \mathbb{R}^{m}$ a set of unquantified parameter variables. Let $F_{i}=f_{i}(X, U) \rho_{i} 0$ for $i=1, \ldots, t$ where $\rho_{i} \in\{=, \geq,>, \neq\} . X_{j}$ is a block of $q_{j}$ quantified variables $(j=1, \cdots, s)$ from $X$ such that $q_{1}+\cdots+q_{s}=n$ and $X_{k} \cap X_{l}=\emptyset$ for all $k$ and $l(k \neq l ; k=1, \ldots, s ; l=$ $1, \ldots, s)$. In general, a first-order formula $\xi$ is described by

$$
\xi=\left({ }^{Q_{1}} X_{1} \ldots{ }^{Q_{s}} X_{s}\right) \tau\left(F_{1}, \ldots, F_{t}\right)
$$

where $\tau\left(F_{1}, \ldots, F_{t}\right)$ is a quantifier-free Boolean formula and $Q_{j} \in\{\forall, \exists\}$.

$\mathrm{QE}$ reduces such a first-order formula to another equivalent formula (i.e., boolean combination of equations/inequalities) without quantified variables. If all variables are quantified, i.e., $m=0, \mathrm{QE}$ decides whether the given formula (12) is true or false. This is a decision problem. When there exist some unquantified variables $U$, QE finds a quantifier-free formula $\zeta(U)$, which is equivalent to $\xi$, describing the regions of admissible $U$ where $\zeta(U)$ is true. Thus the feasible regions are obtained as semi-algebraic sets. If there is no feasible solution, QE outputs "false". For example, a first-order formula $\exists x\left[x^{2}+a x+b \leq c\right]$ is reduced to an equivalent quantifier-free formula $4 c+a^{2} \geq 4 b$ by QE. See [6] for details on QE algorithms. Nowadays there exist several QE implementations on several computer algebra systems, such as QEPCAD [21] on SACLIB, REDLOG [22] on REDUCE, and SyNRAC [3] on Maple. Mathematica has built-in QE commands. It should be noted that $\mathrm{QE}$ computations are executed based on symbolic computation and hence all results are exact.

\section{SOS APPROACH}

We assume that $f$ in system (1) is a polynomial. To rewrite the conditions in Corollary 2.1 as numerically solvable ones, $\alpha_{3}$ and $\alpha_{4}$ are required to be even polynomials that belong to class $\mathcal{K}_{\infty}$ and $\mathcal{K}$, respectively. More precisely, univariate even polynomials strictly increasing in nonnegative region must belong to class $\mathcal{K}_{\infty}$ not class $\mathcal{K}$ [12]. Thus we naturally assume that $\alpha_{4}$ in Corollary 2.1 belongs to class $\mathcal{K}_{\infty}$. The following is a numerically solvable condition that provides a nonlinear gain function of system (1).

Lemma 4.1: [12] he system (1) is input-to-state stable with $\gamma$ if there exist even polynomials $\alpha_{i} \in \mathcal{K}_{\infty}(i=3,4)$, a polynomial $V(x) \in \mathcal{K}_{\infty}$ and an SOS polynomial $s_{0}(x, w)$ such that

$$
\frac{\partial V}{\partial x} f(x, w)-\alpha_{4}(\|w\|)+\alpha_{3}(\|x\|)=-s_{0}(x, w)
$$

for all $(x, w) \in \mathbb{R}^{n} \times \mathbb{R}^{p}$. Then $\gamma(r)=\alpha_{3}^{-1} \circ k \alpha_{4}(r)$ for some $k>1$.

Lemma 4.1 is a convenient tool for searching for an admissible structure of the nonlinear gain function composed of $\alpha_{3}$ and $\alpha_{4}$. Since it is possible to determine unknown coefficients in $\alpha_{3}$ and $\alpha_{4}$, one can decide whether the structure is suitable as nonlinear gain functions. If the corresponding SDP problem is feasible, then the selected structure is suitable and there exist nonlinear gain functions in the structure. For example, we can assume the structures of $\alpha_{3}, \alpha_{4} \in \mathcal{K}_{\infty}$ such as

$$
\begin{aligned}
& \alpha_{3}(r)=b_{1} r^{2}+b_{2} r^{4}+\cdots+b_{s} r^{2 s} \\
& \alpha_{4}(r)=c_{1} r^{2}+c_{2} r^{4}+\cdots+c_{t} r^{2 t}
\end{aligned}
$$

where $b_{i}(i=1,2, \ldots, s)$ and $c_{j}(j=1,2, \ldots, t)$ are nonnegative unknown coefficients. In general, the coefficients are required to satisfy $r \cdot d \alpha_{3}(r) / d r \geq 0$ and $r \cdot d \alpha_{4}(r) / d r \geq 0$ for all $r \in \mathbb{R}$. Such conditions are directly reduced to SOS conditions [12]. Besides, $\alpha_{3}$ and $\alpha_{4}$ cannot have odd power terms of $r$ because $\alpha_{3}(\|x\|)$ and $\alpha_{4}(\|w\|)$ need to be polynomial functions for a direct application of the SOS approach.

Remark 4.1: Lemma 4.1 is available for checking the existence of a selected structure in polynomial functions. 
Such a structure in polynomial functions is also available for the QE approach, which can minimize nonlinear gain functions $\gamma(r)$ for all $r>0$. From an engineering viewpoint, finding a minimum gain function is an important task.

From Lemmas 2.2 and 4.1, a numerically solvable condition has been introduced for the interconnected system (6) and (7).

Lemma 4.2: [12] The interconnected system (6) and (7) is ISS with input $w$ if there exist even polynomials $\alpha_{i j} \in$ $\mathcal{K}_{\infty}, i=3,4, j=1,2, V_{j} \in \mathcal{K}_{\infty}, j=1,2$, sum of squares polynomials $s_{10}\left(x_{1}, x_{2}\right), s_{20}\left(x_{1}, x_{2}\right), s_{30}(r)$ and $s_{40}(r)$ such that

$$
\begin{aligned}
& \frac{\partial V_{1}\left(\left\|x_{1}\right\|\right)}{\partial x_{1}} f_{1}\left(x_{1}, x_{2}\right)-\alpha_{41}\left(\left\|x_{2}\right\|\right)+\alpha_{31}\left(\left\|x_{1}\right\|\right) \\
& =-s_{10}\left(x_{1}, x_{2}\right) \\
& \begin{aligned}
\frac{\partial V_{2}\left(\left\|x_{2}\right\|\right)}{\partial x_{2}} f_{3}\left(x_{1}, x_{2}\right)- & \alpha_{42}\left(\left\|x_{1}\right\|\right)+\alpha_{32}\left(\left\|x_{2}\right\|\right) \\
& =-s_{20}\left(x_{1}, x_{2}\right) \\
\alpha_{32}(r)-\left(1+\varepsilon_{1}\right) \alpha_{41}(r) & =s_{40}(r) \\
\alpha_{31}(r)-\left(1+\varepsilon_{2}\right) \alpha_{42}(r) & =s_{50}(r)
\end{aligned}
\end{aligned}
$$

for all $\left(x_{1}, x_{2}, r\right) \in \mathbb{R}^{n_{1}} \times \mathbb{R}^{n_{2}} \times \mathbb{R}$ where $\varepsilon_{i}>0, i=1,2$.

\section{QE APPROACH}

In the SOS approach, as shown in section IV, the definiteness condition (5) in Corollary 2.1 is replaced by a numerically tractable one (13) in Lemma 4.1 for constructing a nonlinear gain function. Of course, Lemma 4.1 is useful to check whether a gain function exists for a given family of polynomial functions. However, in general, it is hard to accurately verify nonexistence using the SOS approach due to the relaxation gap between (5) and (13).

If the algebraic structures of $V, f, \alpha_{3}$, and $\alpha_{4}$ are given, the original condition (5) can be directly dealt with through $\mathrm{QE}$. This condition is regarded as a $\mathrm{QE}$ problem in a straightforward manner:

$$
(\forall x \forall w)\left[\frac{\partial V}{\partial x} f(x, w)-\alpha_{4}(\|w\|)+\alpha_{3}(\|x\|) \leq 0\right] .
$$

Performing QE on (19), we are able to exactly check the existence/nonexistence of a gain function for given polynomial functions. Furthermore, if feasible solutions to (19) exist, QE provides all feasible solutions for $\alpha_{3}$ and $\alpha_{4}$ (and hence $\gamma$ ). This enables us to find a tight or minimum gain function.

Let us consider functions $\alpha_{3}, \alpha_{4} \in \mathcal{K}_{\infty}$ such as (14) that include unknown coefficients $b_{i}$ and $c_{j}$. If we know in advance that a gain function exists in the structures of these functions, then we can immediately obtain the set on $b_{i}$ and $c_{j}$ without obtaining a "false" output on performing QE on (19). Let $\phi\left(b_{1}, \ldots, b_{s}, c_{1}, \ldots, c_{t}\right)$ be the resulting formula describing the semi-algebraic set on $b_{i}$ and $c_{j}$. Under this set, we can obtain all values of $\alpha_{4}(r)$ for $r>0$ by solving a QE problem:

$$
\begin{aligned}
& \left(\exists b_{1} \cdots \exists b_{s} \exists c_{1} \cdots \exists c_{t}\right) \\
& \quad\left[\alpha_{3}(z)=\alpha_{4}(r) \wedge \phi\left(b_{1}, \ldots, b_{s}, c_{1}, \ldots, c_{t}\right)\right] .
\end{aligned}
$$

Let $\psi(r, z)$ be the resulting formula defining the set on $r$ and $z$. Further let $z^{*}=z^{*}(r)$ be the lower boundary polynomial of the feasible set given by $\psi(r, z)$; this polynomial is called a bottom curve on $\psi(r, z)$. Then $\underline{\gamma}(r)=z^{*}$ is the bottom curve on $\psi(r, z)$.

Remark 5.1: In fact the QE approach is very powerful, but unfortunately, computational complexity of QE is (doubly) exponential [6]. In the problems (19) and (20), it is desirable to not produce "false" on performing QE. If QE performed on the problem (19) outputs "false," then we need to select the structures in $\alpha_{3}$ and $\alpha_{4}$ again. That is, the computational cost is added to the doubly exponential complexity. On the other hand, for example, the problem (20) always has a feasible set $\psi(r, z)$ if the problem (19) produces a set $\phi\left(b_{1}, \ldots, b_{s}, c_{1}, \ldots, c_{t}\right)$.

Remark 5.2: In the case where the gain function is a solution of an algebraic equation of the fifth or higher degree, in general, it is not possible to describe the gain as an explicit form, or an algebraic expression.

\section{SOS-QE APPROACH}

In this section, we propose a hybrid procedure using SOS and $\mathrm{QE}$ to analyze nonlinear gain functions of the systems. This procedure is then demonstrated using a system.

The following is the procedure:

Step 1 Select a candidate polynomial Lyapunov function $V \in \mathcal{K}_{\infty}$, even polynomials $\alpha_{3}, \alpha_{4} \in \mathcal{K}_{\infty}$ where $\alpha_{3}$ and $\alpha_{4}$ include unknown coefficients $b_{i}(i=$ $1,2, \ldots, s)$ and $c_{j}(j=1,2, \ldots, t)$, respectively.

Step 2 Solve the SOS problem (13). If it does not have a feasible solution, then return to Step 1.

Step 3 Solve the QE problem (19) and obtain all the sets on $b_{i}(i=1,2, \ldots, s)$ and $c_{j}(j=1,2, \ldots, t)$, i.e., $\phi\left(b_{1}, \ldots, b_{s}, c_{1}, \ldots, c_{t}\right)$.

Step 4 Solve the QE problem (20) and obtain all the set on $r$ and $z$, i.e., $\psi(r, z)$. Pick up the bottom curve $z^{*}=z^{*}(r)$ that minimizes $z$ for $r(>0)$. Then $\underline{\gamma}(r)$ is given by $z^{*}$ for the selected structures in $\alpha_{3}$ and $\alpha_{4}$.

Remark 6.1: Information on a specific problem is very important for reducing computational complexity while performing QE. Since computational time for Step 2 is much less than that for Step 3, Step 2 is very meaningful.

Remark 6.2: In the case where $\alpha_{3}(r)$ is given, it is possible to minimize $\alpha_{4}(r)$ for a fixed $r$ in the problem formulation in the SOS problem (13). For example, we can consider an SOS optimization problem:

$$
\left.\underset{\substack{c_{1} \geq 0, \ldots, c_{t} \geq 0 \\ s_{0}(\text { is SOS })}}{\min } \alpha_{4}(r)\right|_{r=1} \text { s.t. }(\forall x \forall w)[(13)]
$$

However, problem (21) does not fit our purpose because (21) minimizes $\alpha_{4}(r)$ only on $r=1$ while we need to minimize $\alpha_{4}(r)$ on each $r>0$. In the case where both $\alpha_{3}(r)$ and $\alpha_{4}(r)$ are unknown, a similar optimization scheme is difficult to perform by SOS. 
To illustrate the above procedure, we have a few examples. We use a PC with Intel Core i5-460M CPU $(2.53 \mathrm{GHz})$ and 8 GB memory. SOS problems are computed on Matlab R2010a with YALMIP(R20101208) and SeDuMi(ver.1.3) while QE problems are computed on Mathematica 8.

\section{A. Example 1}

Consider a polynomial nonlinear system in [15]:

$$
\dot{x}=-x-2 x^{3}+\left(1+x^{2}\right) w^{2} .
$$

Now set a candidate Lyapunov function $V(x)=x^{2} / 2 \in \mathcal{K}_{\infty}$, which is a part of Step 1 in the procedure. The structures of $\alpha_{3}$ and $\alpha_{4}$ are selected as

$$
\alpha_{3}(r)=r^{2}, \quad \alpha_{4}(r)=c_{1} r^{2}+c_{4} r^{8}
$$

where $c_{1}$ and $c_{4}$ are nonnegative numbers. Then one can solve an SOS problem in Step 2 and obtain a feasible pair $\left(c_{1}, c_{4}\right)=(1.5255,1.4578)$. A computational time for solving the SDP problem is 0.4 [s]. Here we note that an optimization of the SOS problem gives us $\left(c_{1}, c_{4}\right)=$ $(0.4407,0.1273)$ with $\alpha_{4}(1)=0.5680$. We will review this fact at the end of this example. In Step 3, eliminating $x$ and $w$ in the formula (19), we obtain the set described by $\phi\left(c_{1}, c_{4}\right)$ shown in Fig.1 (left upper). A computational time is 1.1 [s]. One can also see both of the pairs $\left(c_{1}, c_{4}\right)$ from the SOS problems. On the basis of $\phi\left(c_{1}, c_{4}\right)$, we solve the QE problem in Step 4, and obtain a feasible set on $r$ and $z$, or $\psi(r, z)$, which is shown in Fig.1 (right upper). Finally, one can find a minimum gain function as

$$
\gamma(r)=z^{*}(r)
$$

where $z^{*}$ is obtained in Step 4. The left lower in Fig.1 shows the gain functions from a QE optimal (solid), an SOS optimal (dotted) at $r=1$ and a SOS feasible (dashed). As we have noted, the SOS optimal agrees with the QE optimal at $r=$ 1. Similarly, in the right lower part, an SOS optimization (dotted) at $r=2$ is indicated. One can see that our final result minimizes the gain function in all $r>0$.

\section{B. Example 2}

Consider a polynomial nonlinear system in [15]:

$$
\dot{x}=-(1+w) x^{3}-x^{5} .
$$

In Step 1, we choose a candidate Lyapunov function $V(x)=$ $x^{2} / 2$ again, and

$$
\alpha_{3}(r)=b_{3} r^{6}, \quad \alpha_{4}(r)=r^{2}+c_{3} r^{6}
$$

where $b_{3}$ and $c_{3}$ are nonnegative numbers. In Step 2, we find a feasible pair $\left(b_{3}, c_{3}\right)=(0.2379,0.0443)$ from an SOS problem. In a similar way as the previous example, we obtain the set $\psi(r, z)$ in Step 4. These results are shown in Fig.2. A result of the gain structure by letting $\alpha_{3}(r)=b_{2} r^{4}$ and $\alpha_{4}(r)=r^{2}+c_{2} r^{4}$ is shown in the right lower. In this example, a remarkable difference between the two gain structure remains.
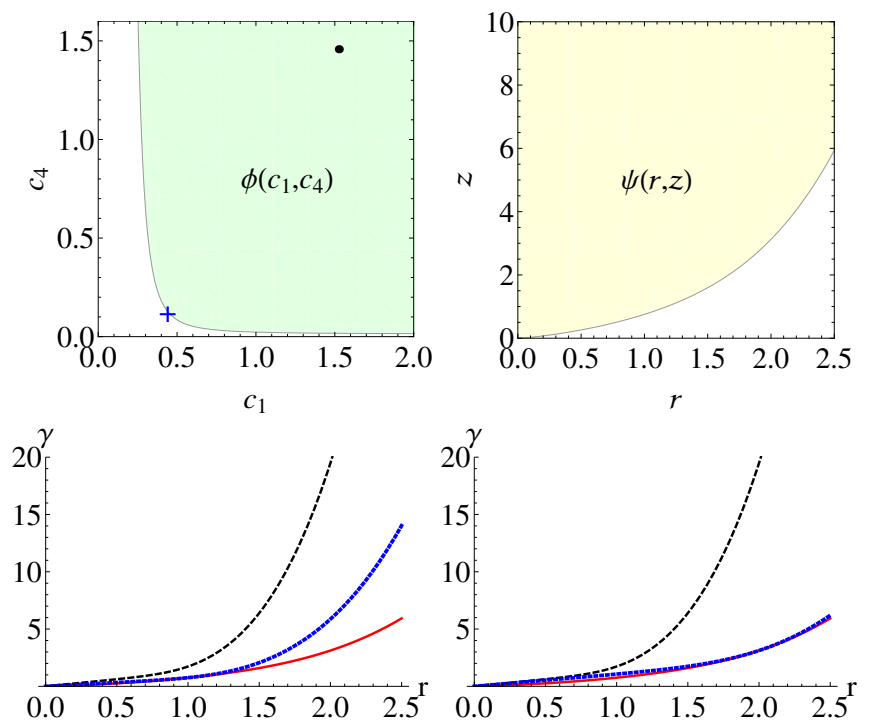

Fig. 1. Sets defined by $\phi\left(c_{1}, c_{4}\right)$ (left upper) and $\psi(r, z)$ (right upper), and gain functions (lowers)
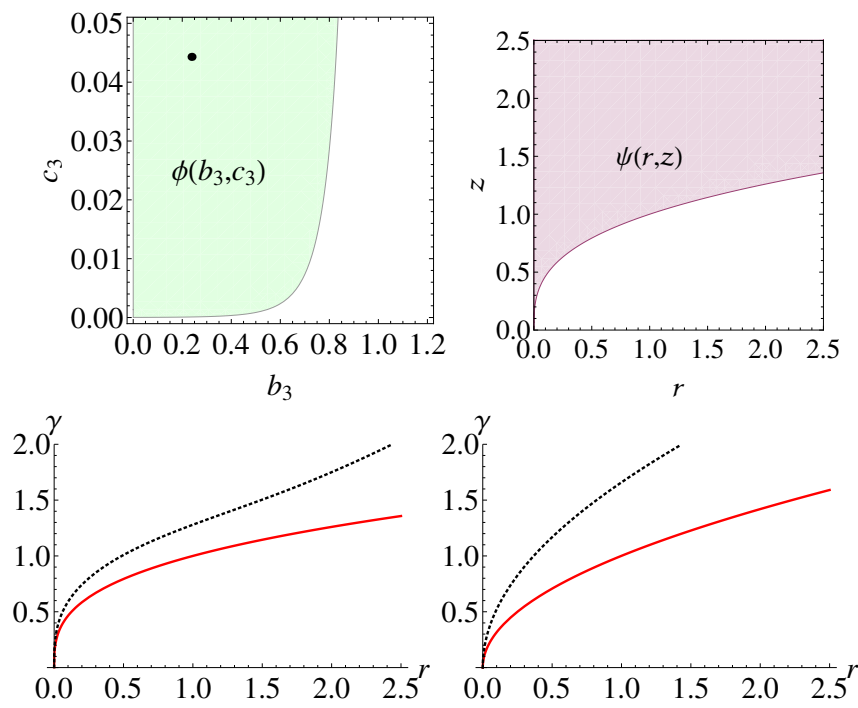

Fig. 2. Sets defined by $\phi\left(b_{3}, c_{3}\right)$ (left upper), $\psi(r, z)$ (right upper) and a gain function (left lower). A gain function with other structure (right lower).

\section{Example 3}

Consider the interconnected system in [23]

$$
\begin{aligned}
& \dot{x}_{1}=-x_{1}+x_{2}^{2} \\
& \dot{x}_{2}=-a_{1} x_{2}^{3}+a_{2} x_{1} x_{2}
\end{aligned}
$$

where $a, b \in \mathbb{R}$. We will reveal a region of $\left(a_{1}, a_{2}\right)$ that the interconnected system is 0-GAS using the hybrid procedure with Lemmas 2.2 and 4.2. In Step 1, each of the SOS problems in Lemma 4.2 are solved for a fixed pair $\left(a_{1}, a_{2}\right)=$ $\left(1, \frac{1}{2}\right), V_{1}\left(x_{1}\right)=x_{1}^{2}$ and $V_{2}\left(x_{2}\right)=x_{2}^{2}$. The resulting gain functions are

$$
\begin{array}{ll}
\alpha_{31}(r)=0.9402 r^{2}, & \alpha_{41}(r)=1.2094 r^{4}, \\
\alpha_{32}(r)=1.4060 r^{4}, & \alpha_{42}(r)=0.7328 r^{2} .
\end{array}
$$

The $x_{1}$-subsystem is ISS with input $x_{2}$ and the nonlinear gain function is $\gamma_{1}(r)=\alpha_{31}^{-1} \circ \alpha_{41}(r)=1.1342 r^{2}$, and 
the $x_{2}$-subsystem is ISS with input $x_{1}$ and the nonlinear gain function is $\gamma_{2}(r)=\alpha_{32}^{-1} \circ \alpha_{42}(r)=0.8497 r^{\frac{1}{2}}$. Thus $\gamma_{1} \circ \gamma_{2}(r)=0.8188 r<r$ and the interconnected system is 0-GAS. Then we apply Step 2-4 to each subsystems and obtain the gain functions as follows:

$$
\gamma_{1}(r)=r^{2}, \gamma_{2}(r)=\left(\frac{a_{2}^{2} r^{2}}{-1+2 a_{1}}\right)^{1 / 4} \text {. }
$$

Finally, solving the QE problem

$$
(\forall r)\left[r>0 \Rightarrow \gamma_{1} \circ \gamma_{2}(r)<r\right]
$$

we obtain a set that the interconnected system is 0-GAS as

$$
\begin{gathered}
\left\{a_{2} \neq 0 \wedge a_{1}>\frac{1+a_{2}^{2}}{2}\right\} \vee\left\{a_{2}=0 \wedge a_{1} \neq \frac{1}{2}\right\} . \\
\text { VII. CONCLUSION }
\end{gathered}
$$

The new procedure of computing a nonlinear gain function has been proposed for nonlinear polynomial systems by a hybrid use of the SOS and QE approaches. The QE approach can find all the possible gain functions in a given structure and can minimize the functions for all magnitude of the input while the SOS approach gives us a structure of the function. The new procedure expands a range of application for nonlinear gain analysis.

\section{APPENDIX}

\section{GAIN FUNCTION IN EXAMPLE 1}

An explicit form of the gain function $\gamma(r)$ is

$$
\left(\frac{256 r^{4}+9 r^{8}}{2048}+\frac{663552 r^{8}+41472 r^{12}+729 r^{16}}{55296 a(r)}+\frac{3 a(r)}{2048}\right)^{\frac{1}{2}}
$$

where

$$
\begin{aligned}
& a(r)=\left(8388608 r^{8}+1048576 r^{12}+69632 r^{16}\right. \\
& +2304 r^{20}+27 r^{24}+4096\left(4194304 r^{16}\right. \\
& \left.\left.+1048576 r^{20}+102400 r^{24}+4864 r^{28}+112 r^{32}+r^{36}\right)^{\frac{1}{2}}\right)^{\frac{1}{3}} .
\end{aligned}
$$

\section{GAIN FUNCTION IN EXAMPLE 2}

An explicit form of the gain function $\gamma(r)$ is

$$
\begin{aligned}
& \frac{1}{3^{\frac{2}{3}} \sqrt{2}}\left(648 r^{2}+4901 r^{6}\right. \\
& +(-145262358703+2422282644 \sqrt{3873})^{\frac{1}{3}} r^{6} \\
& \left.-(145262358703+2422282644 \sqrt{3873})^{\frac{1}{3}} r^{6}\right)^{\frac{1}{6}}
\end{aligned}
$$

\section{REFERENCES}

[1] P. Dorato, W. Yang, and C. Abdallah, "Robust multi-objective feedback design by quantifier elimination," J. Symb. Comput., vol. 24, no. 2, pp. 153-159, 1997.

[2] N. Hyodo, M. Hong, H. Yanami, S. Hara, and H. Anai, "Solving and visualizing nonlinear parametric constraints in control based on quantifier elimination," Appl. Algebra Eng. Commun. Comput., vol. 18, no. 6, pp. 497-512, 2007.

[3] H. Yanami and H. Anai, "The maple package SyNRAC and its application to robust control design," Future Generation Comp. Syst., vol. 23, no. 5, pp. 721-726, 2007.

[4] H. Anai, "A symbolic-numeric approach to multi-parametric programming for control design," in Proc. ICROS-SICE International Conferece, 2009, pp. 3525-3530.
[5] H. Yanami, "Quantifier elimination and its application to optimization problems in engineering," Ph.D. dissertation, Kyushu University, 2010.

[6] B. F. Caviness and J. R. Johnson, Eds., Quantifier Elimination and Cylindrical Algebraic Decomposition, ser. Texts and monographs in symbolic computation. Springer-Verlag, 1998.

[7] P. A. Parrilo, "Structured semidefinite programs and semialgebraic geometry methods in robustness and optimization," Ph.D. dissertation, California Institute of Technology, Pasadena, CA, may 2000.

[8] G. Chesi, A. Garulli, A. Tesi, and A. Vicino, "Solving quadratic distance problems: an LMI-based approach," IEEE Trans. on Automatic Control, vol. 48, no. 2, pp. 200-212, 2003.

[9] S. Boyd, L. E. Ghaoui, E. Feron, and V. Balakrishman, Linear Matrix Inequalities in Systems and Control Theory. Philadelphia: SIAM Studies in Applied Mathematics, 1994.

[10] E. D. Sontag and Y. Wang, "On characterizations of the input-to-state stability property," Systems \& Control Letters, vol. 24, no. 4, pp. 351359, 1995.

[11] A. Isidori, Nonlinear Control Systems II. Springer, 1999.

[12] H. Ichihara, "Computational approach to input-to-state stability analysis of a class of nonlinear systems," in Proc. ICROS-SICE International Conferece, 2009, pp. 3914-3918.

[13] H. Ichihara and H. Anai, "A symbolic-numeric approach to nonlinear gain analaysis of a class of dynamical systems," in Proc. of 2010 IEEE Multi-Conf. on Systems and Control, 2010, pp. 1708-1713.

[14] Z. P. Jiang, A. R. Teel, and L. Praly, "Small-gain theorem for ISS systems and applications," Mathematics of Control, Signals and Systems, pp. 95-120, 1994.

[15] H. K. Khalil, Nonlinear Systems, 3rd edition. Prentice Hall, 2001.

[16] S. Prajna, A. Papachristodoulou, P. Seiler, and P. A. Parrilo, SOSTOOLS: Sum of squares optimization toolbox for MATLAB, 2004. [Online]. Available: http://www.cds.caltech.edu/sostools

[17] J. Löfberg, "YALMIP : A toolbox for modeling and optimization in MATLAB," in Proc. of the CACSD Conf., Taipei, Taiwan, 2004, available from http://control.ee.ethz.ch/joloef/yalmip.php.

[18] J. F. Sturm, SeDuMi: Using sedumi 1.02, a matlab toolbox for optimization over symmetric cones (updated for version 1.05), 1998. [Online]. Available: http://sedumi.ie.lehigh.edu/

[19] K. C. Toh, R. H. Tütüncü, and M. J. Todd, On the implementation and usage of SDPT3 - a Matlab software package for semidefinitequadratic-linear programming, version 3.0, 2001. [Online]. Available: http://www.math.cmu.edu/ reha/sdpt3.html

[20] D. Henrion and A. Garulli, Positive Polynomials in Control, LNCIS Vol.312. Springer, 2005.

[21] C. W. Brown, "QEPCAD B - a program for computing with semialgebraic sets using cads," SIGSAM Bull., vol. 37, no. 4, pp. 97-108, 2003.

[22] A. Dolzmann and T. Sturm, "REDLOG: computer algebra meets computer logic," SIGSAM Bull., vol. 31, no. 2, pp. 2-9, 1997.

[23] H. Ito, "Characterizing systems with stability from a nonlinear view point: a primary concept and application of input-to-state stability (in japanese)," Journal of the Society of Instrument and Control Engeers, vol. 42, no. 10, pp. 789-795, 2003. 